\title{
Chancen (und Risiken) der Digitalisierung
}

\author{
Jean Martin \\ Dr. med., Mitglied der Redaktion
}

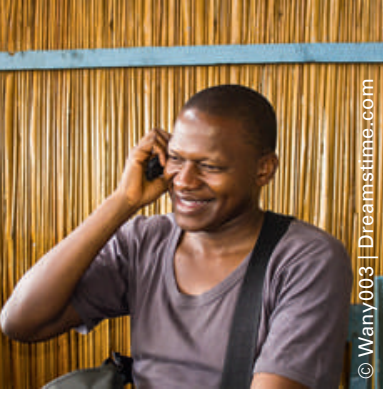

Auch in Afrika setzt man viel auf die Mobilfunktechnologie.

\footnotetext{
Literatur

1 Martin J. Madagascar, l'Ile rouge, en une quinzaine d'instantanés. Bulletin des médecins suisses. 2012;93: 1879-82.

2 Partnerschaft. (Geschäftsstelle Helvetas, 3001 Bern). August 2016, Nr. 225, 13-22.

3 Thöni Th. Novartis se lance dans la révolution Healthcare 4.0. 24 heures (Lausanne), 30 août 2016, p.11.

4 Kiefer B. Le nouveau monde de la recherche clinique. Revue médicale suisse. 2016;12:1400
}

Die digitale Revolution verändert unsere Welt in unglaublicher Geschwindigkeit. Unsere (Enkel-)Kinder scheinen damit keine Probleme zu haben, wenngleich sich wohl die Frage stellt, ob die Physiologie des Menschen - und im weiteren Sinne auch die Gesellschaft als Ganzes, unsere Kultur - flexibel genug ist, um mit dieser Geschwindigkeit "Schritt halten» zu können. In diesem Kontext ist das Mobiltelefon ein besonders gutes Beispiel. Auf Reisen erstaunte mich seine Omnipräsenz. "Madagaskar ist ein armes Land, aber alle scheinen auf diese Technologie zu setzen. So ist beispielsweise die Tatsache, dass Geld 'auf' das Mobiltelefon geladen und damit Verbindlichkeiten im Handel getilgt werden können, ein wertvoller Service, um Diebstahl und Betrug zu vermeiden» [1].

Helvetas widmet der digitalen Entwicklung eine Ausgabe ihres Magazins [2]. Nirgendwo wächst die Anzahl der Mobiltelefonnutzer so schnell wie in Afrika. Ein Ansprechpartner aus Mali meint dazu: «Eigentlich dachten wir, die Mittelklasse würde sich auf das Gerät stürzen. Aber es waren die Handwerker, Marktverkäuferinnen und LKW-Fahrer.» Als der Norden des Landes 2012-2013 von islamistischen Rebellen besetzt wurde, «wurden die Radiostationen von den Mobiltelefonnutzern mit [sehr wertvollen] Informationen beliefert [...]. Bei den Wahlen ist das Mobiltelefon ebenfalls ein wichtiges Werkzeug im Kampf gegen Wahlbetrug.» So kann Information trotz Zensur und Unterdrückung zirkulieren.

Die Behörden nutzen die Mobiltelefonie, um ihre Bürger über verschiedene Themen zu informieren, u.a. in Notfällen, wie bei der Ebola-Epidemie. "Auch die Ausbildung wird völlig verändert. Was bei Schulbüchern oft Jahre dauert, wird nun in den elektronischen Medien in Echtzeit realisiert.» Besteht die Gefahr, dass sich eine Kluft auftut zwischen jenen, die über ein Mobiltelefon verfügen, und jenen, die keines haben? «Der digitale Zugang ist deutlich grösser und umfassender, als dies bei den alten Technologien der Fall war. In Afrika haben 600 Millionen Menschen keinen Zugang zur Stromversorgung, aber sieben von zehn Personen verfügen über ein Mobiltelefon.» Es fand eine unglaubliche Mutation statt: Laut dem Helvetas-Magazin verlief die Kommunikation zwischen der Schweiz und ihren Partnerländern bis zum Jahr 1980 über den
Postweg. Inzwischen geschieht dies einfach per Klick. Beschrieben werden auch IT-Projekte in Bosnien eines in der Ausbildung und ein weiteres in der Tourismusförderung. Der durch IT ermöglichte Austausch ist ein wertvolles Instrument zur Sensibilisierung für verbreitete soziale Probleme - beispielsweise die Misshandlung von Frauen - und zum Kampf und zur Vorsorge in diesen Bereichen.

Das Phänomen ist global; es ist geographisch umfassend und betrifft alle gesellschaftlichen Aktivitäten. Es bietet Chancen, weckt aber auch Ängste, vor allem in Bezug auf den Schutz der Privatsphäre. Jeder Mobiltelefonnutzer kann lokalisiert werden! Dies ist von Vorteil, wenn man allein in den Bergen unterwegs ist (gelegentlich passiert mir das ohne Natel und ich halte es heute für kritisierbar), aber eben nicht immer.

In der Medizin, u.a. in der Epidemiologie oder in der Public Health, gibt uns die Digitalisierung Mittel und Wege an die Hand, um komplexe Verhältnisse besser und schneller erfassen und verstehen zu können. Big Data ermöglicht die Bearbeitung riesiger Datenmengen, ihre Verknüpfung mit vielen Variablen, das Aufzeigen von Korrelationen in einer Art und Weise, wie dies zuvor nicht denkbar war. Die Pharmaindustrie ist natürlich damit beschäftigt: Novartis unterstützt die Einrichtung weltumfassender Datennetze zum Thema Gesundheit und führt Telefon-Apps für Patienten ein [3]. Bertrand Kiefer fragt in einem seiner jüngsten Artikel nach der Forschung und sagt: "In den Wissenschaften sind Daten inzwischen - ohne Rücksicht auf alte Machtstrukturen - dabei, die Medizin zu revolutionieren. Um diese Daten wird jedoch gefeilscht und gehandelt [...] Digitale Medizin muss daher von einem ethischen $\mathrm{Pa}-$ radigmenwechsel begleitet sein. Die in der Bevölkerung erfassten Gesundheitsdaten sind als Teil des 'Gemeingutes' der Menschheit zu betrachten. Dieses 'Gemeingut' zählt zu den Schätzen der Welt und hat keine Besitzer. All dies ist bedroht» [4]. Heute ist dies klar ein grosses Problem: Was menschliches Gemeingut sein und von der Menschheit (inkl. den kommenden Generationen) geteilt werden sollte, wird häufig durch Privatisierung zu lukrativen Zwecken in Frage gestellt. 